\title{
Enhanced 'In-situ' Catalysis via Microwave Selective Heating: Catalytic Chain Transfer Polymerisation
}

\author{
Kevin Adlington, ${ }^{\mathrm{a}, \mathrm{b}}$ Robert McSweeney, ${ }^{\mathrm{b}}$ Georgios Dimitrakis, ${ }^{\mathrm{a}}$ Samuel W. \\ Kingman, ${ }^{\mathrm{a}}$ John P. Robinson, ${ }^{\mathrm{a}}$ Derek J. Irvine ${ }^{\mathrm{a}, \mathrm{b}^{*}}$ \\ ${ }^{a}$ National Centre for Industrial Microwave Processing, Process and \\ Environmental Research Division, Faculty of Engineering, and ${ }^{\mathrm{b}} \mathrm{School}$ of \\ Chemistry, University of Nottingham, Nottingham, NG7 2RD, UK.
}

\begin{abstract}
An extremely facile, single stage, 'in-situ', Catalytic Chain Transfer Polymerisation (CCTP) process has been identified, where the optimal polymerisation process was shown to depend upon a combination of catalyst characteristics (i.e. solubility, sensitivity, activity) and the method of heating applied. In comparison to the current benchmark catalyst, the preparation of which is only about $40 \%$ efficient, this represents a significant increase in waste prevention/atom efficiency and removes the need for organic solvent. It was also shown possible to significantly reduce the overall 'in-situ' reaction cycle time by adopting different processing strategies in order to minimise energy use. The application of microwave heating was demonstrated to overcome system diffusion/dilution issues and result in rapid, 'in-situ' catalyst formation. This allowed processing times to be minimised by enabling a critical concentration of the species susceptible to microwave selective heating to dominate the heat and mass transfer involved.
\end{abstract}

\section{Introduction}

Many modern material applications now require polymers of relatively low molecular weight (Mwt) to successful deliver the desired performance in their 
intended use, e.g. surfactants, dispersants, emulsifiers, high solid organic coatings, etc. ${ }^{1,2,3,4}$ This has led to the development of new chain transfer/ control systems which enable the successful manufacture of lower Mwt polymers in high yield. In the specific field of free radical polymerisation (FRP), catalytic chain transfer polymerisation (CCTP) has proven to be a very efficient method of achieving the controlled synthesis of such low Mwt polymers. Furthermore, CCTP has an additional advantage in addressing the needs of the application types detailed above, many of which require materials that are amphiphillic in nature. This is because CCTP generates polymers that are exclusively terminated by a vinylic functionality (see Figure 1). This allows these oligomeric materials to be used to construct amphiphillic materials in a subsequent chemical transformation with monomeric/polymeric species containing a different/desired functionality in one of 3 ways.

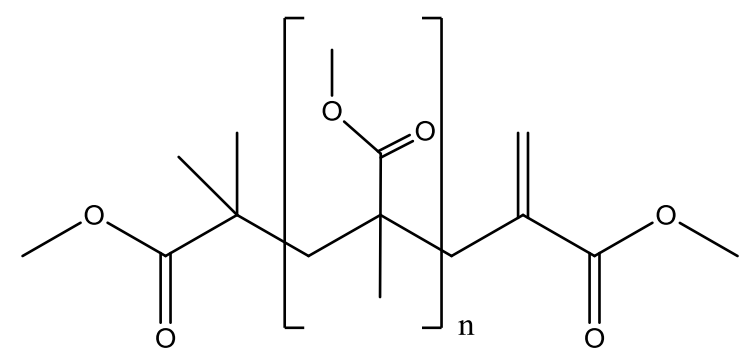

Figure 1. General molecular structure of CCTP oligomers of methyl methacrylate (MMA).

These are: (a) acting as a single event chain transfer agent via a $\beta$-scission mechanism which introduces an element of the oligomers differing molecular character onto both the terminated and initiated chains that result, (b) acting as a macromonomer in the production of branched copolymers by being incorporated into the backbone of a material in a subsequent polymerisation 
with a monomer containing a second functionality and/or (c) being endfunctionalised at the double bond to add an alternative functionality. $5,6,7,8$

In practice the CCTP technique utilises certain low spin Co(II) complexes, which have been demonstrated to act as catalytic chain transfer agents (CTAs), to achieve a far greater level of control over the Mwt of the final polymer product, when compared to the more conventional single event CTAs (e.g. thiols)..$^{9,10,11}$ It is because of the catalytic nature of the CCTP control agents that they exhibit greatly enhanced rates of chain transfer to polymer. They can undergo the necessary termination/reinitiation process many times, whilst a thiol can achieve this only once. Consequently, the application of CCT agents is a facile way to achieve; (a) the isolation of polymers with very low Mwt's, (b) targeted Mwt polymers using very low CTA concentrations, and/or (c) a reduction in undesired properties of the reaction mixture, such as odour or colour, due to the low concentration of CTA used..$^{9,10,11}$

The most widely accepted mechanism for CCTP proposes a two-step process. In step $1\left(\mathrm{R}_{\mathrm{n}}+\mathrm{Co}(\mathrm{II}) \rightarrow \mathrm{P}_{\mathrm{n}}+\mathrm{Co}(\mathrm{III})-\mathrm{H}\right)$ a growing polymer chain $\left(\mathrm{R}_{\mathrm{n}}\right)$ interacts with the $\operatorname{Co}(\mathrm{II})$ complex which results in a dead polymer chain $\left(\mathrm{P}_{\mathrm{n}}\right)$ and formation of a $\mathrm{Co}(\mathrm{III})$ hydride species which is thought to be the rate limiting step. During step 2 (Co(III)-H + monomer $\rightarrow \mathrm{R}_{1}+\mathrm{Co}(\mathrm{II})$ ) this $\mathrm{Co}(\mathrm{III}) \mathrm{H}$ intermediate reacts with a monomer to form a new monomeric radical $\left(\mathrm{R}_{1}\right)$ and reform the $\mathrm{Co}(\mathrm{II})$ catalyst. ${ }^{11,12}$

One of the most commonly cited catalysts used to achieve reproducible CCTP is bis[(difluoroboryl) diphenylglyoximato]cobalt(II) (PhCoBF) which is synthesised via a two-step process. The first involves the reaction of cobalt (II) acetate $\left(\mathrm{Co}(\mathrm{Ac})_{2}\right)$ with two equivalents of diphenylglyoxime (dpg) under inert 
conditions. $^{12}$ The product complex from this stage (cobaloxime) has been shown to be an active CCTP catalyst, but one which is sensitive to deactivation by either/both hydrolysis and oxidation. ${ }^{13,14}$ The second step involves the reaction of the stage 1 product with $\mathrm{BF}_{3} . \mathrm{OEt}_{2}$, generating a single tetradentate ligand from the two bidentate dpg ligands in cobaloxime.${ }^{14}$ This delivers both enhanced activity and significantly improved stability towards oxidation and hydrolysis, allowing PhCoBF to be handled readily in air in solid form.

In a recent publication by the authors, an improvement in the atom efficiency, sustainability and potential industrial applicability of CCTP processes was reported. ${ }^{15}$ This was delivered by successfully achieving in a single step, both the direct, 'in-situ' synthesis of low spin Co complexes (see Figure 2) and the subsequent CCTP control of a polymerisation of methyl methacrylate (MMA) using this 'in-situ' CTA, when operated as a bulk, solution and expanded phase based process.

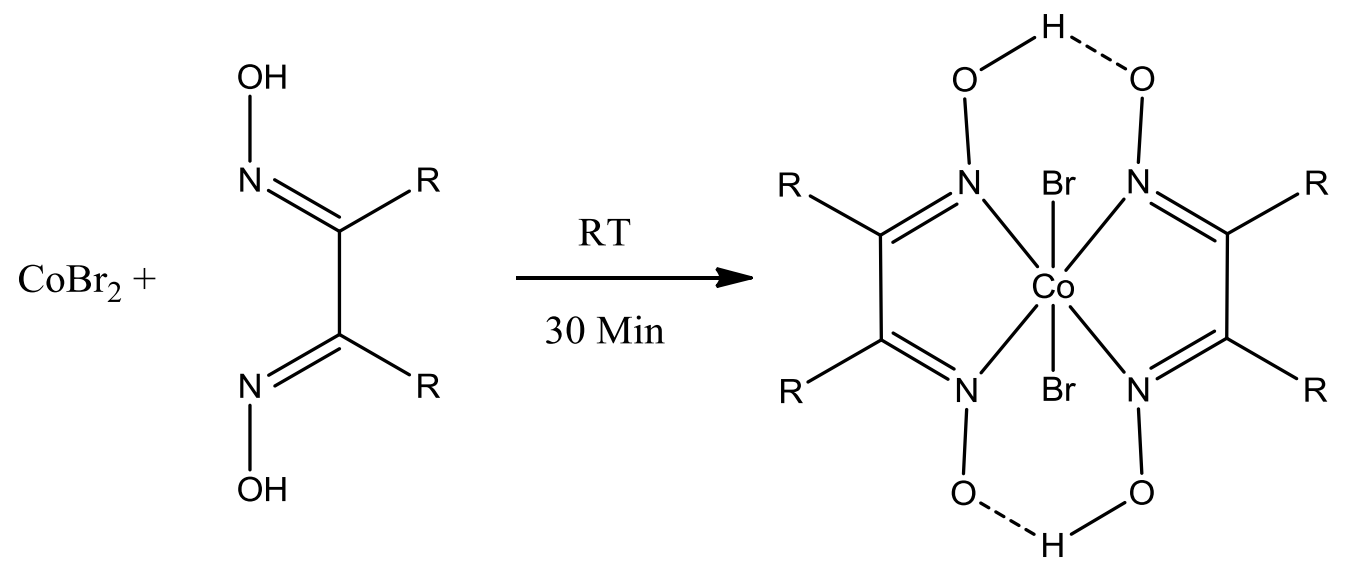

Figure 2. Reaction of $\mathrm{CoBr}_{2}$ and the relevant equatorial ligand to form the active catalyst

Adopting these CTA's removed the need for the inefficient and problematic reaction with $\mathrm{BF}_{3} \cdot \mathrm{Et}_{2} \mathrm{O}$, a reagent which is toxic, highly air/moisture sensitive and pyrophoric. Furthermore, it was shown that this 'in-situ' methodology was 
actually more efficient than pre-preparing and isolating the CCTP agents. This increase in efficiency was attributed to avoiding catalyst degradation related to isolation and storage. However, this work demonstrated that significant prestirring regimes were required to achieve a high quality target product, especially when low catalyst concentrations were utilised. These factors significantly reduced the commercial viability of an industrial scale process based on this methodology.

This paper details optimisation of the process efficiency and large scale viability of this 'in-situ' CCTP processes by investigating the effect of accelerating and harmonising the rates of 'in-situ' catalyst generation and polymerisation by the use of selective microwave heating. There have been a number of such combined reaction rate and process efficiency improvements reported in the literature to date, where the overall value of the microwave heating has been realised by either conducting the polymerisation with reduced/no solvent, ${ }^{16,17}$ or in alternative solvents such as ionic liquids. ${ }^{18,19}$ The aims of this study were to define if adopting this heating method, which has been shown to accelerate catalyst formation in other systems, ${ }^{20}$ would make it possible to (a) remove the need for a lengthy pre-stir prior to starting the polymerisation and (b) improve Mwt control at low catalyst concentrations.

\section{Experimental}

Materials:- Anhydrous cobalt bromine (99 \%, Aldrich), cobalt acetate tetrahydrate (99\%, Aldrich) dimethyl and diphenylglyoxime (93\%, Aldrich), and $\mathrm{PhCoBF}$ (Dupont) were all used as supplied without further purification. MMA (99 \%, Aldrich) was passed through a column of basic alumina to 
remove the inhibitors prior to use. The 2,2-Azobis(isobutyronitrile) (AIBN, 98\%, Aldrich) was purified by being recrystallized twice from methanol. All organic solvents were dried and degassed with argon prior to use.

Reactor Geometries:- All microwave heated (MWH) reactions were conducted in a CEM Discover microwave reactor (maximum power $300 \mathrm{~W}$ operating at 2.45GHz) equipped with an IR temperature sensor. An additional optical fibre (OF) temperature sensor was introduced directly into the reaction bulk via the use of a septum and a cylindrical microwave choke fitted to the top of the reactor. This allows access to the vessel whilst keeping the microwave energy contained within the reactor to levels below that required by Health and Safety legislation. Comparative and conventionally heated $(\mathrm{CH})$ experiments were conducted using a standard oil bath, where the oil temperature was controlled via a thermocouple inserted in the heating fluid and the actual bulk temperature was monitored by use of the OF probe. In this way the "true" bulk temperature of the $\mathrm{CH}$ reaction medium was measured and cross referenced to both that of the heating fluid and the comparative MWH experiments OF probe bulk temperature measurement.

Characterisation:- Gel Permeation Chromatography (GPC) Analysis:- GPC was performed on a Polymer Labs GPC-120 instrument at $40{ }^{\circ} \mathrm{C}$ equipped with a PLgel $5 \mu \mathrm{m}$ guard column and two $30 \mathrm{~cm}$ PolarGel-M Columns in series coupled with a refractive index detector using HPLC grade THF as the mobile phase at a flow of $1.0 \mathrm{~cm}^{3} \cdot \mathrm{min}^{-1}$. The GPC was calibrated with poly(methyl methacrylate) narrow PDI standards ranging from $690-1,944,00 \mathrm{~g} \cdot \mathrm{mol}^{-1}$. All 
GPC equipment and standards were supplied by Polymer Laboratories (Varian). GPC data were analysed using the Cirrus GPC Offline software package. For the low molecular weight materials produced, the standard deviation of the GPC measurements calculated from 10 individual measurements was defined to be $\pm 50 \mathrm{~g} \cdot \mathrm{mol}^{-1}$.

NMR Analysis $-{ }^{1} \mathrm{H}\left(\delta_{H}\right)$ NMR spectra:- were recorded at $25{ }^{\circ} \mathrm{C}$ using a Bruker DPX-300 spectrometer $(300 \mathrm{MHz})$ and chemical shifts were recorded in $\delta_{\mathrm{H}}$ (ppm). Samples are prepared as solutions in $\mathrm{CDCl}_{3}$ to which chemical shifts were referenced (residual chloroform at $7.26 \mathrm{ppm}$ ). Analysis of the spectra was carried out using ACDLABS 12 software. The ${ }^{1} \mathrm{H}$ NMR $\left(300 \mathrm{MHz}, \mathrm{CDCl}_{3}, 25\right.$ $\left.{ }^{\circ} \mathrm{C}\right)$ for MMA and CCTP derived PMMA are as follows: MMA. $\delta \mathrm{H} 3.70(3 \mathrm{H}$, $\left.\mathrm{CH}_{3}-\mathrm{O}\right), 6.07\left(1 \mathrm{H}, \mathrm{C}=\mathrm{CH}_{2}\right), 5.57\left(1 \mathrm{H}, \mathrm{C}=\mathrm{CH}_{2}\right), 1.90\left(3 \mathrm{H}, \mathrm{CH}_{3}\right)$. CCTP oligomers of PMMA $\delta_{\mathrm{H}} 3.64\left(6 \mathrm{H}, \mathrm{CH}_{3}-\mathrm{O}\right), 6.29\left(1 \mathrm{H}, \mathrm{C}=\mathrm{CH}_{2}\right), 5.79(1 \mathrm{H}$, $\left.\mathrm{C}=\mathrm{CH}_{2}\right), 2.63\left(2 \mathrm{H}, \mathrm{CH}_{2}=\mathrm{CCH}_{2}\right), 1.17\left(6 \mathrm{H}, 2 \mathrm{CH}_{3}\right)$. To calculate the conversion for the NMR data, the size of the integral exhibited by the oligomer/polymer resonance located at $3.64 \mathrm{ppm}$ was ratioed against the resonance for the monomer located at $3.7 \mathrm{ppm}$. This figure was then multiplied by 100 to give the overall conversion as a percentage figure.

Combined Gas Chromatography/Mass Spectral Analysis (GC-MS):- GC-MS was performed on a VG/Micromass/ Waters AutoSpec in EI+mode. A $15 \mathrm{~m}$ capillary column of type BP-1 of $0.25 \mathrm{~mm}$ thickness was used, with helium at $80 \mathrm{kPa}$ employed as the carrier gas. Typically, $50 \mathrm{mg}$ of sample was dissolved in $1 \mathrm{~mL}$ of dichloromethane for analysis and the parameters used for the GC 
analysis of compounds in dichloromethane were: column temperature was taken from room temperature to $50{ }^{\circ} \mathrm{C}$ in three minutes, then raised up to 280 ${ }^{\circ} \mathrm{C}$ by $4{ }^{\circ} \mathrm{C} \min ^{-1}$; injection temperature was $250{ }^{\circ} \mathrm{C}$; sample size was $1 \mathrm{~mL}$.

Mayo calculations for PhCoBF:- A stock solution of PhCoBF was prepared by dissolving PhCoBF (22 mg, $0.0348 \mathrm{mmol})$ in MMA (25 ml, $235 \mathrm{mmol})$. A second initiator stock solution was prepared by dissolving AIBN (300 mg, 1.83 mmol) in MMA (150 ml, $1410 \mathrm{mmol})$. Five reaction mixtures were then prepared, each containing $25 \mathrm{~mL}$ of the initiator solution and a mixture of MMA and the PhCoBF stock solution totalling $5 \mathrm{~mL}$. The amounts of $\mathrm{PhCoBF}$ stock solution used in the five reactions were $0,0.5,1.0,2.0$, and $4.0 \mathrm{ml}$. After being deoxygenated by three cycles of freeze pump thaw and sealed, all five reactions were stirred simultaneously at $60{ }^{\circ} \mathrm{C}$ for $15 \mathrm{~min}$ prior to being quenched in ice. After being quenched in ice the monomer was evaporated from the reaction solution. GPC was carried out on the entire reaction mixture to avoid fractionation on precipitation. Yields were determined by ${ }^{1} \mathrm{H}$ NMR

CCTP Procedures:- All stock solutions and polymerisation reactions were prepared/conducted under an argon atmosphere using standard Schlenk techniques, unless stated otherwise. All polymerisations were conducted without application of freeze pump thaw techniques being adopted in the preparation of the reaction mixtures. All calculations were corrected for reagent purity. In detailing the polymerisation procedures, in each case, typical reactions for a single halide are presented. Furthermore, the data presented are typical values representing at least five repeat experiments, the typical standard 
deviation founded in heating times were $+/-1$ minute. In the assessment of the molecular weight of the final products the typical standard deviation was found to be $+/-50 \mathrm{~g} \mathrm{~mol}^{-1}$ which was supported by the close agreement between the proton NMR and GPC estimation of molecular weight.

All the CCTP polymerisations included in this paper with the exception of 2 (Table 5, Entries 5 and 6) have used the same level of initiator and reaction temperature. Thus they would be expected to generate the same Mwt product polymer if no CTA were added. Therefore, the corresponding blank experiment common to all the CCTP has been included as Table 1, Entry 1.

Additionally, attempts were made to follow the "in-situ" formation of the catalysts using UV/Vis analysis. However, these attempts did not generate results which were repeatable therefore it proved impossible to obtain representative data. This was attributed to a combination of the low catalyst concentrations involved and variable levels of degradation of the catalysts during sampling and analysis.

General Synthesis of DiHalide $\left(\mathrm{dmgH}_{2}\right)_{2}$ cobalt and DiHalide $\left(\mathrm{dpg} \mathrm{H}_{2}\right)_{2}$ cobalt :Dimethylglyoxime $\mathrm{DmgH}_{2}(1.6 \mathrm{~g}, 13.6 \mathrm{mmol})$ or diphenylglyoxime (3.2 g, $13.6 \mathrm{mmol})$ was added to a stirred solution of cobalt(II) halide hydrate (1.50 g, $6.8 \mathrm{mmol}$ ) in $50 \mathrm{~mL}$ of acetone. After $30 \mathrm{~min}$, the solution was cooled on ice, filtered, and washed $(2 \times 15 \mathrm{~mL}$ acetone $)$ giving a fine powder. Yields were obtained gravimetrically and catalyst purity was assessed using UV/Vis measurements, $\lambda \max ($ MMA $)=496 \mathrm{~nm}$. The compounds exhibited very low solubility and was therefore not characterised spectroscopically. 
General Polymerisation techniques adopted with pre-prepared powdered catalysts without pre-stirring:- For a typical polymerisation using pre-prepared PhCoBF, PhCoBF (0.0169 g, $0.0377 \mathrm{mmol})$ and AIBN (0.141 g, $0.859 \mathrm{mmol}$ ) were added to a Schlenk tube and degassed under argon for 30 minutes, following this pre-degassed MMA $(30 \mathrm{ml}, 280 \mathrm{mmol})$ was transferred to the Schlenk tube under an argon atmosphere. The solution was then heated in a thermostated oil bath to $80{ }^{\circ} \mathrm{C}$ for two hours. Termination of the reaction involved rapid cooling of the reaction vessel upon removing it from the oil bath, to ensure that reaction mixture remained representative of the reaction's progress at that time. The solution was then precipitated into an excess of hexane (typically $1 \mathrm{~L}$ ) and the product polymer retrieved by filtration. If no precipitate was retrieved, the solvents were removed from the solution by use of a rotator evaporator to isolate the product MMA oligomer as oils. The product oligomers was then dried for 7 days in a vacuum oven $\left(25^{\circ} \mathrm{C}, 10^{-1}\right.$ mbar) and the product was then analysed by GPC and ${ }^{1} \mathrm{H}$ NMR following the method described above.

Polymerisation with an in-situ DiHalide $\left(\mathrm{dmgH}_{2}\right)_{2}$ cobalt catalyst without prestirring:- Cobalt(II) halide hydrate $(0.0174 \mathrm{~g}, 0.0796 \mathrm{mmol})$, AIBN (0.141 g, $0.859 \mathrm{mmol})$ and $\mathrm{dmgH}_{2}(0.0184 \mathrm{~g}, 0.159 \mathrm{mmol})$ were added to a Schlenk tube and degassed under argon for 30 minutes. Following this, pre-degassed MMA (30 $\mathrm{ml}, 280 \mathrm{mmol}$ ) was transferred to the Schlenk tube under an argon atmosphere. The solution was heated in a thermostated oil bath to $80{ }^{\circ} \mathrm{C}$ for two hours. Termination of the reaction involved rapid cooling of the reaction vessel upon removing it from the oil bath to ensure that reaction mixture 
remained representative of the reaction's progress at that time. The solution was then precipitated into an excess of hexane (typically $1 \mathrm{~L}$ ) and the product polymer retrieved by filtration. If no precipitate was retrieved, the solvents were removed from the solution by use of a rotator evaporator to isolate the product MMA oligomer as oils. The product oligomers was then dried for 7 days in a vacuum oven $\left(25^{\circ} \mathrm{C}, 10^{-1} \mathrm{mbar}\right)$ and the product was then analysed by GPC and ${ }^{1} \mathrm{H}$ NMR as described above.

Polymerisation with an in situ DiHalide $\left(\mathrm{dmgH}_{2}\right)_{2}$ cobalt catalyst with stirring for 30 minutes at room temperature:- Cobalt(II) halide hydrate $(0.0174 \mathrm{~g}$, $0.0796 \mathrm{mmol})$, AIBN $(0.141 \mathrm{~g}, 0.859 \mathrm{mmol})$ and $\mathrm{dmgH}_{2}(0.0184 \mathrm{~g}, 0.159$ mmol) were added under argon to a degassed solution of MMA (30 ml, 280 mmol) contained in s schlenk tube with an inert atmosphere. The contents were then stirred for 30 mins at room temperature. Following this, a solution of AIBN (0.141g, $0.859 \mathrm{mmol})$ dissolved in a solution of degassed MMA (5 ml, 46.4 mmol) was added under argon and the solution was heated in a thermostated oil bath to $80{ }^{\circ} \mathrm{C}$ and stirred at that temperature for two hours. Termination of the reaction and isolation of the product polymer was then conducted as described in the general polymerisation procedure.

General experimental procedure for microwave heated reactions:- The reagent preparation procedure was as described above for the $\mathrm{CH}$ reactions. However, these reactions were conducted in the CEM Discover microwave reactor system described above, by introducing heat energy via exposure to microwaves. The reaction vessel and contents, prepared as described above, 
were heated to $80{ }^{\circ} \mathrm{C}$ by being inserted into the cavity of the CEM rather than oil bath. The internal average bulk temperatures were continually measured during the application of the microwave energy, using both an optical fibre (OF) probe inserted directly into the reaction mixture and the CEM's external infra-red (IR) sensor. The combination of these measurements allowed the power input to be controlled at a level required to keep the bulk temperature at the target set point of $80{ }^{\circ} \mathrm{C}$.

\section{Results and Discussion}

As discussed above, the current benchmark CCTP agent/catalyst (PhCoBF) exhibits an attractive balance between stability and activity. However, because the preparation of $\mathrm{PhCoBF}$ involves an inefficient two stage synthetic process, the overall catalyst costs can becomes significant when it is considered for use in full scale industrial manufacture. ${ }^{14}$ Meanwhile, the only major drawback to an 'in-situ' method of catalyst manufacture was increased reaction cycle times. ${ }^{15}$ This was due to the inclusion of a 1 hour pre-stir to ensure that the catalyst had been synthesised prior to commencing the polymerisation.

Thus, the initial reactions in this programme were conducted to evaluate -the levels of pre-stirring that was required for a range of halide ligands as the previous work had only investigated performance of brominated complexes. MMA was used as the test monomer, and polymerisations were conducted in the bulk to maximise cobalt CTA activity and involved the use of both dpg and dmg to additionally define if a less sterically bulky equatorial ligand would further optimise the 'in-situ' process. ${ }^{9}$ Complexing dmg, produces MeCoBF, the molecular structure of which is similar to PhCoBF but contains 4 methyl 
ligand substituents. Finally, the 1 hour pre-stir adopted in the previous publication was reduced to 30 minutes to define if this was sufficient to synthesise the catalyst before the polymerisation process began. ${ }^{15}$ The results of the experiments which varied the halide ligand with dmg can be seen in Table 1.

Table 1. Polymer data for bulk CCTP of MMA using $600 \mathrm{ppm}$ of 'in-situ' dmg catalyst, $0.5 \mathrm{wt} \%$ AIBN for 2 hours at $80^{\circ} \mathrm{C}$ with $30 \mathrm{~min}$ pre-stir at RT

\begin{tabular}{cccccc}
\hline Entry & $\begin{array}{c}\text { Axial } \\
\text { Ligand }\end{array}$ & $\begin{array}{c}\text { Equatorial } \\
\text { Ligand }\end{array}$ & $\begin{array}{c}\text { Conversion } \\
(\mathbf{\%})^{\mathbf{a}}\end{array}$ & PDI & $\begin{array}{c}\text { Mn } \\
\left(\mathbf{g ~ m o l}^{-1}\right)^{\mathbf{b}}\end{array}$ \\
\hline $\mathbf{1}^{\mathbf{c}}$ & - & - & 97 & 2.50 & 54,000 \\
$\mathbf{2}$ & $\mathrm{Br}$ & $\mathrm{dmg}$ & 35 & 2.08 & 1,200 \\
$\mathbf{3}$ & $\mathrm{Cl}$ & $\mathrm{dmg}$ & 83 & 2.36 & 2,100 \\
$\mathbf{4}$ & $\mathrm{I}$ & $\mathrm{dmg}$ & 89 & 2.59 & 3,000 \\
\hline
\end{tabular}

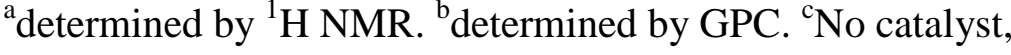

In the absence of a CTA (Table 1, Entry 1) at $80{ }^{\circ} \mathrm{C}$, both a high conversion (97 $\%$ ) and a high $\mathrm{M}_{\mathrm{n}}$ polymer (54,000 $\left.\mathrm{g} \mathrm{mol}^{-1}\right)$ were obtained. However, when the reaction was conducted using $\mathrm{CoBr}_{2}$ and $\mathrm{dmg}$ as catalyst precursors (Table 1, Entry 2) (See ESI - Figure S1 for NMR), with the same initiator concentration and a pre-stir at RT, the Mwt was noted be significantly lower $\left(1,200 \mathrm{~g} \mathrm{~mol}^{-1}\right)$. This was concluded to be a clear indication that the CTA is both forming insitu' and then successfully controlling the Mwt. In comparison, when $\mathrm{CoCl}_{2}$ was the cobalt containing catalyst precursor (Table 1, Entry 3), a decrease in Mwt was noticed but it was found to be less pronounced and the PDI was also observed to be higher. It was postulated that this was because the $\mathrm{Cl}$ ligand had a higher charge density, thus it may be less likely to undergo the reductive elimination step necessary to form the active catalyst. Dong et al. reported Raman spectroscopy investigating Co-R bonds which found that the larger the substituent the weaker the bond, supporting the proposal that the $\mathrm{Cl}$ complex 
would be less likely to undergo reductive elimination. ${ }^{21}$ Similar findings were obtained when Jensen et al. reported density functional theory (DFT) on vitamin $\mathrm{B}_{12}$, they also found that larger substituents resulted in weaker bonds. ${ }^{22}$ However, 'in-situ' polymerisations which utilised $\mathrm{CoI}_{2}$ as the precursor were noted to produce the smallest reduction in Mwt (Table 1, Entry 4). This latter result was attributed to the larger iodine substituent increasing the steric crowding around the cobalt disfavouring the complexation of the dmg ligand which effectively reduces the amount of active catalyst present reaction. This conclusion was also supported by the observation that the PDI is greater suggesting that more of the polymerisation has occurred in the presence of reduced levels of catalyst. Thus, whilst all halide precursors did actively participate in the 'in-situ' CCTP process, the bromide precursor was concluded to deliver the optimum results.

The next process variable investigated were the influence of pre-synthesis, isolation and addition of the halide catalyst to the reaction. The results of these experiments are detailed in Table 2.

Table 2. CCTP of MMA in bulk using 600ppm (a) pre-synthesised and (b) 'insitu' dmg catalyst and $0.5 \mathrm{wt} \%$ AIBN for 2 hours at $80{ }^{\circ} \mathrm{C}$ with 30 mins prestirring at RT

\begin{tabular}{cccccc}
\hline Entry & $\begin{array}{c}\text { Axial } \\
\text { Ligand }\end{array}$ & Method & $\begin{array}{c}\text { Conversion } \\
(\mathbf{\%})^{\mathbf{a}}\end{array}$ & PDI & $\begin{array}{c}\mathbf{M n} \\
\left(\mathbf{g ~ m o l}^{-\mathbf{1}}\right)^{\mathbf{b}}\end{array}$ \\
\hline $\mathbf{1}$ & $\mathrm{Br}$ & Pre-sythn & 62 & 2.28 & 1,400 \\
$\mathbf{2}$ & $\mathrm{Br}$ & 'in-situ' & 35 & 2.08 & 1,200 \\
$\mathbf{3}$ & $\mathrm{Cl}$ & Pre-sythn & 73 & 2.86 & $\sim 25,000$ \\
$\mathbf{4}$ & $\mathrm{Cl}$ & 'in-situ' & 83 & 2.36 & 2,100 \\
$\mathbf{5}$ & $\mathrm{I}$ & Pre-sythn & 80 & 2.29 & 4,200 \\
$\mathbf{6}$ & $\mathrm{I}$ & 'in-situ' & 89 & 2.59 & 3,000 \\
\hline${ }^{\text {a }}$ determined by ${ }^{1}$ H NMR. $^{\text {b }}$ determined by GPC
\end{tabular}

When $\mathrm{CoBr}_{2}$ and $\mathrm{CoI}_{2}$ were reacted with dmg they both generated an isolated 
catalyst (green and brown/black powders respectively) which still controlled the reaction of MMA. However as previously reported with the dpg liganded catalysts ${ }^{15}$ the molecular weight of the isolated polymer indicated that neither exhibited as effective control as when used in the 'in-situ' method. Figure 3 is an example of the comparative GPC traces for the 'in-situ' and pre-synthesised polymers produced using $\mathrm{CoBr}_{2}$. (See ESI - Figure $\mathrm{S} 2$ for $\mathrm{CoI}_{2} \mathrm{GPC}_{\text {Overlay). }}$

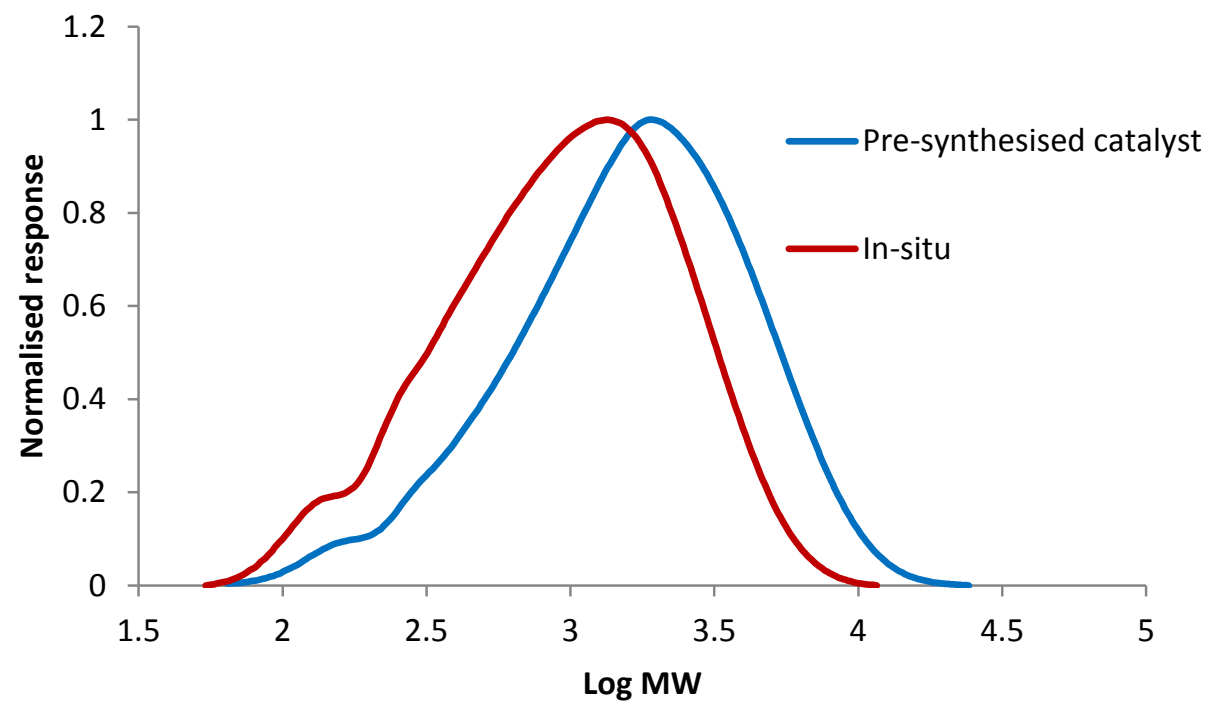

Figure 3. Comparison of the GPC traces of the product polymer synthesised using $\mathrm{CoBr}_{2}$ and dmg in (a) 'in-situ' and (b) pre-synthesised catalyst reactions

However, when the same reactions were carried out with $\mathrm{CoCl}_{2}$ the molecular weight was observed to be significantly lower for the 'in-situ' catalyst when compared with the $\mathrm{CoCl}_{2}$ pre-synthesised reaction (see Table 2, Entries 3 and 4). This was because the isolated $\mathrm{CoCl}_{2}(\mathrm{dmg})_{2}$ complex was much more air sensitive than the bromo- and iodo- equivalents. It was visibly observed to degrade from the original blue complex recovered immediately upon precipitation, to a CCTP inactive purple complex over the course of an hour. Therefore, this reaction highlighted the efficiency of the 'in-situ' process because it allows this unstable complex to be formed and actively participate in 
the chain transfer cycle. It also presents a differing explanation for the order of activity exhibited by the halide catalyst. The fact that the chloride gives a final product with slightly increased molecular weights may simply be due to its greater sensitivity resulting in increased deactivation compared the $\mathrm{Br}$ equivalent. This conclusion was supported by the fact that unlike the GPC's for the $\mathrm{CoBr}_{2}$ and $\mathrm{CoI}_{2}$ polymers that of the $\mathrm{CoCl}_{2}$ data was found to be bimodal indicating that there is catalyst loss during the reaction and thus a move to higher molecular weight as the reaction proceeds (see Figure 4).

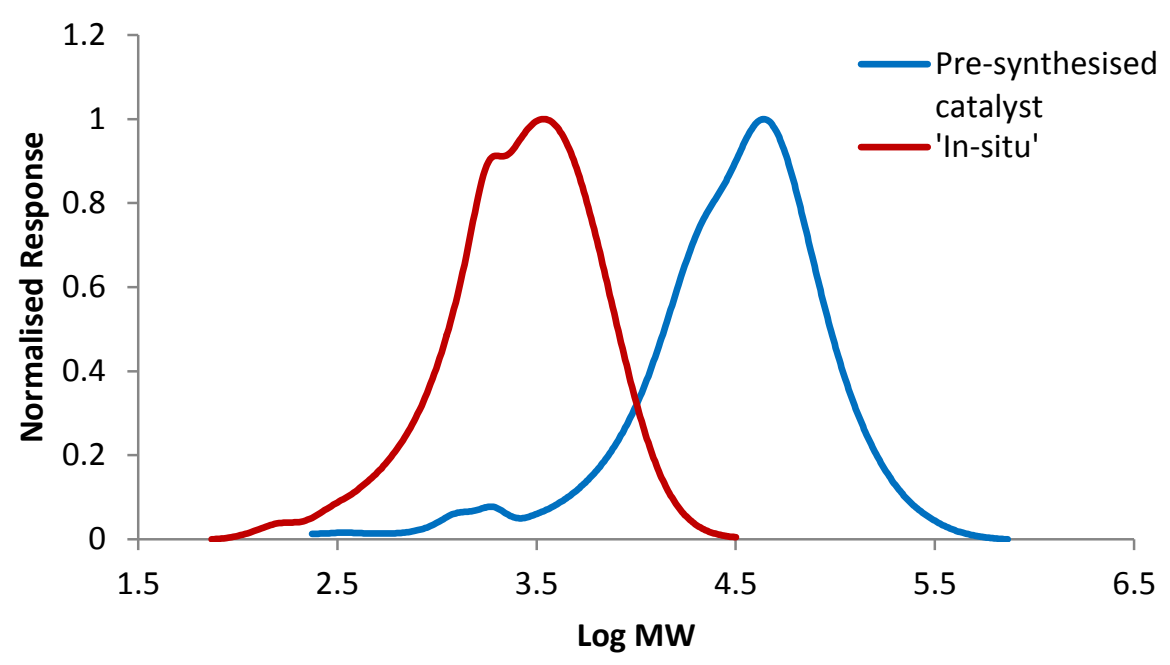

Figure 4. Comparison of the GPC traces of the product polymer synthesised using $\mathrm{CoCl}_{2}$ and dmg in (a) 'in-situ' and (b) pre-synthesised catalyst reactions

Therefore, it was concluded that adopting $\mathrm{Cl}$ and $\mathrm{I}$ axial ligands did not increased the efficiency of the pre-synthesis route. Rather, all the halide complexes were shown to be more effective in the 'in-situ' process.

The influence of the time taken for catalyst formation was then investigated. Up to this point the polymerisation procedures had all included 30 minute prestirring prior to the addition of the initiator to allow for CCTP agent manufacture. The results in Table 3 show the influence of omitting the pre- 
stirring stage.

Table 3. CCTP of MMA in bulk using 600ppm of 'in-situ' dmg catalyst and 0.5 wt $\%$ AIBN for 2 hours at $80{ }^{\circ} \mathrm{C}$ with no pre-stirring

\begin{tabular}{cccccc}
\hline Entry & $\begin{array}{c}\text { Axial } \\
\text { Ligand }\end{array}$ & Method & $\begin{array}{c}\text { Conversion } \\
(\boldsymbol{\%})^{\mathbf{a}}\end{array}$ & PDI & $\begin{array}{c}\text { Mn } \\
\left(\mathbf{g ~ m o l}^{\mathbf{1}}\right)^{\mathbf{b}}\end{array}$ \\
\hline $\mathbf{1}$ & $\mathrm{Br}$ & 'in-situ' & 72 & 2.20 & 900 \\
$\mathbf{2}$ & $\mathrm{Cl}$ & 'in-situ' & 71 & 2.09 & 1,300 \\
$\mathbf{3}$ & $\mathrm{I}$ & 'in-situ' & 69 & 2.24 & 2,100 \\
\hline
\end{tabular}

${ }^{a}$ determined by ${ }^{1}$ H NMR. ${ }^{b}$ determined by GPC

Interestingly, all the polymerisations which were conducted without prestirring were observed to produce polymer with lower molecular weights. For example, in the $\mathrm{CoBr}_{2}$ case, removing the pre-stir delivered a reduction of the molecular weight from 1,200 $\mathrm{g} \mathrm{mol}^{-1}$ to $900 \mathrm{~g} \mathrm{~mol}^{-1}$ (Table 3, Entry 1). These results indicated that not only was the pre-stirring of the reaction not necessary to achieve control over the polymerisation at the $600 \mathrm{ppm}$ loading, but it has been demonstrated to actually reduce the systems efficiency. It was also noted that the order of catalyst activity was the same either with or without prestirring Therefore, the proposed reason for these observations is that the active catalyst is being formed more rapidly when pre-stirring is applied, thus it has a greater length of time to react with any residual air/moisture/protic impurities that might be present in the system leading to increased deactivation of the catalyst.

Now that the principle of the 'in-situ' method had been demonstrated with the dmg ligand, a comparative series of experiments adopting dpg as the equatorial ligand were conducted. These produced single stage equivalents to $\mathrm{PhCoBF}$, and so investigated the effect on the 'in-situ' control imparted by the increased solubility and stability, but reduced activity of the phenyls 
complexes. The results of these experiments are detailed in Table 4.

Table 4. CCTP of MMA in bulk using 600ppm of 'in-situ' dpg catalyst and 0.5 wt $\%$ AIBN for 2 hours at $80^{\circ} \mathrm{C}$ with no pre-stirring

\begin{tabular}{cccccc}
\hline Entry & $\begin{array}{c}\text { Axial } \\
\text { Ligand }\end{array}$ & $\begin{array}{c}\text { Equatorial } \\
\text { Ligand }\end{array}$ & $\begin{array}{c}\text { Conversion } \\
(\mathbf{\%})^{\mathbf{a}}\end{array}$ & PDI & $\begin{array}{c}\text { Mn } \\
\left(\mathbf{g ~ m o l}^{\mathbf{1}}\right)^{\mathbf{b}}\end{array}$ \\
\hline $\mathbf{1}$ & $\mathrm{Br}$ & $\mathrm{dpg}$ & 35 & 1.34 & 300 \\
$\mathbf{2}$ & $\mathrm{Cl}$ & $\mathrm{dpg}$ & 43 & 1.89 & 500 \\
$\mathbf{3}$ & $\mathrm{I}$ & $\mathrm{dpg}$ & 58 & 2.19 & 700 \\
$\mathbf{4}^{\mathbf{c}}$ & - & - & 32 & 1.18 & 200 \\
\hline${ }^{\text {a }}$ determined by ${ }^{1} \mathrm{H}$ NMR. & ${ }^{\text {b }}$ determined by GPC. ${ }^{\text {c }}$ reaction uses PhCoBF \\
instead of 'in-situ' method. & & &
\end{tabular}

With the dpg ligand the same activity order was observed for the 3 halide precursors. Additionally, with no pre-stirring of the reaction were observed to deliver a further decrease in both PDI and molecular weight when compared to the dmg products. However a significant drop in yield was also observed. For example, in the case of the $\mathrm{CoBr}_{2}$ precursor the molecular weight of the product polymer was observed to decrease from $900 \mathrm{~g} \mathrm{~mol}^{-1}$ to $300 \mathrm{~g} \mathrm{~mol}^{-1}$ on moving to the dpg ligand (comparison of Table 3, Entry 1 with Table 4, Entry 1), which is similar to the molecular weight obtained when using presynthesised PhCoBF (Table 4, Entry 4). This is in line with the data published in a previous paper by the authors which determined the chain transfer constant (Cs) by using the Mayo equation method. ${ }^{15,23,24}$ The Cs value of the $\mathrm{CoBr}_{2}$ 'in-situ' system was determined to be in the region of 8,000 to 10,000 which compares favourably with the literature reported $\mathrm{Cs}$ for $\mathrm{PhCoBF}$ of the 17,000 - 20,000 at $60{ }^{\circ} \mathrm{C}$ in MMA. ${ }^{9}$ By comparison the $\mathrm{Cs}$ of most Thiol based industrially applied standard CTA are of the order of 1.0.

The reduction in conversion with dpg compared to dmg was attributed to a combination of the following factors; (a) the greater solubility of the phenyl 
complexes and (b) their reduced sensitivity hence reduced deactivation. Both of these lead to a greater quantity of catalyst being present in the reaction solution which both increased the molecular weight reduction observed and the level of retardation that CCTP introduces to a FRP reaction at high catalyst concentrations because a high percentage of the radicals actively involved in the catalytic cycle at a specific unit time.

In this case a comparison of the PDI's is invalid because the distribution of the lower molecular weight oligomers generated by the $\mathrm{PhCoBF}$ and the $\mathrm{CoBr}_{2}$ precursor merge with that the residual monomer truncating the data. Consequently, GC-MS analysis was conducted on these samples to definitively demonstrate that these catalysts are indeed producing mixtures of dimer and trimer. Figure 6 details this GC-MS data, which clearly shows that the main products of the 'in-situ' reaction are MMA dimer and trimer and a small amount of tetramer. Furthermore, the balance of the quantities of oligomers present in the final reaction mixture was comparable to that obtained by $\mathrm{PhCoBF}$ to within the error of the measurement technique/apparatus used.

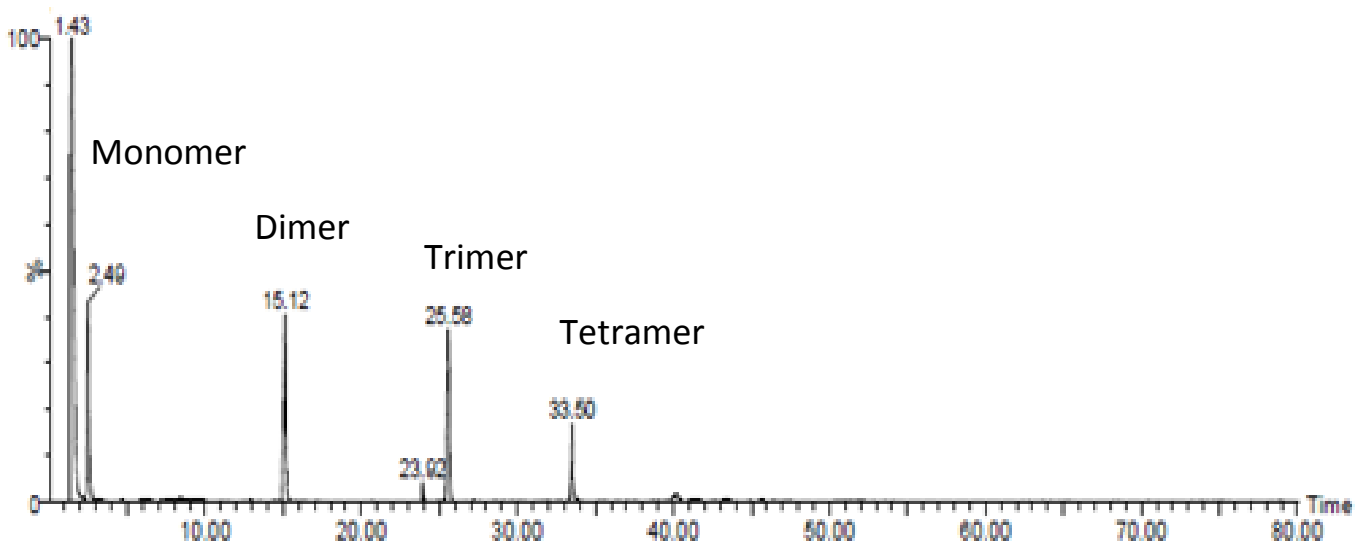

Figure 6 GC-MS of the MMA bulk reaction using $600 \mathrm{ppm}$ of the CCTP agent generated from the reaction of $\mathrm{CoBr}_{2}$ and $\mathrm{dpg}$. 
Thus the conclusion from the above data was that the optimum catalyst in terms of molecular weight reduction observed was that isolated from $\mathrm{CoBr}_{2}$ and dpg, However, it has clearly been confirmed by this study that a significant elongation of reaction time is observed with the quantity of catalyst required. It also needs to be confirmed that as the catalyst concentration is reduced the 'insitu' method still remains competitive with the pre-synthsised PhCoBF benchmark. The latter may result in the catalyst formation being significantly slowed due to the additional dilution of the precursors.

Thus the potential to address both of these drawbacks by applying microwave heating was investigated. It has been demonstrated by the authors that the application of volumetric heating can significantly increase the rate of CCTP reactions, in some cases reducing them from hours to minutes. ${ }^{16}$ Additionally the authors have also recently reported that 'in-situ' organometallic reactions can be severely reduced in time, even at high dilution by the influence of selective heating. ${ }^{20}$

The initial reactions investigated the potential to reduce the reaction time of the optimum $\mathrm{CoBr}_{2} / \mathrm{dpg}$ system and the results of these are detailed in Table 5 .

Table 5. CCTP of MMA in bulk using 600ppm of $\mathrm{CoBr}_{2}$ 'in-situ' catalyst at 80 ${ }^{\circ} \mathrm{C}$ adopting microwave heating and with no pre-stirring

\begin{tabular}{cccccccc}
\hline $\begin{array}{c}\text { Heating } \\
\text { Method }\end{array}$ & $\begin{array}{c}\text { Equatorial } \\
\text { Ligand }\end{array}$ & $\begin{array}{c}\text { [AIBN] } \\
(\mathbf{w t} \%)\end{array}$ & $\begin{array}{c}\text { Time } \\
(\mathbf{m i n})\end{array}$ & $\begin{array}{c}\text { Conv } \\
(\mathbf{\%})^{\mathbf{a}}\end{array}$ & PDI & $\begin{array}{c}\text { Mn } \\
\left(\mathbf{g ~ m o l}^{-\mathbf{1}}\right)^{\mathbf{b}}\end{array}$ \\
\hline $\mathbf{1}$ & $\mathrm{CH}$ & $\mathrm{dpg}$ & 0.5 & 120 & 35 & 1.34 & 300 \\
$\mathbf{2}$ & $\mathrm{MWH}$ & $\mathrm{dpg}$ & 0.5 & 120 & 35 & 1.39 & 300 \\
$\mathbf{3}$ & $\mathrm{CH}$ & $\mathrm{dmg}$ & 0.5 & 120 & 72 & 2.20 & 900 \\
$\mathbf{4}$ & $\mathrm{MWH}$ & $\mathrm{dmg}$ & 0.5 & 120 & 81 & 2.31 & 800 \\
$\mathbf{5}$ & $\mathrm{CH}$ & $\mathrm{dpg}$ & 1.0 & 120 & 47 & 2.50 & 200 \\
$\mathbf{6}$ & $\mathrm{MWH}$ & $\mathrm{dpg}$ & 1.0 & 3 & 31 & 1.29 & 200 \\
\hline${ }^{\mathrm{a}}$ determined by ${ }^{\mathrm{I}} \mathrm{H} \mathrm{NMR.}^{\mathrm{b}}$ determined by GPC & & &
\end{tabular}


These data further exemplify the conclusion previously reported by the authors. ${ }^{16}$ In all cases, where $0.5 \mathrm{wt} \%$ AIBN was applied, the exotherrm was well controlled and thus there is no difference observed in the $\mathrm{CH}$ and $\mathrm{MWH}$ data. Thus the results are essentially the same in (Table 5, Entries 1 and 2) both conversion and Mwt. Thus, if conducted in this way there appears no advantage in using MWH, However, with 1 wt $\%$ AIBN (Table 5, Entries 5 and 6) the reaction quickly exotherms to $100{ }^{\circ} \mathrm{C}$ in the microwave only. Thus the reaction is terminated after only 3 minutes to keep the bulk temperature below the boiling point of the monomer. This exotherm has been previously reported by the authors and attributed to the onset of volumetric, selective heating of the free radicals present. ${ }^{16}$

To confirm that it was the increase in initiator concentration which is responsible for this microwave induced increase in yield, comparative $\mathrm{CH}$ and MWH experiments were conducted at the lower $0.5 \mathrm{wt} \%$ initiator level with the dmg ligand. This complex had been shown to deliver less control and thus retardation to the system. In practice, a significant exotherm was observed in both of these reactions via the optical fibre temperature probe inserted into the bulk of the reaction medium. The temperature in both was found to rise to 90 $95{ }^{\circ} \mathrm{C}$ and took $\sim 25$ minutes to stabilise at the target $80{ }^{\circ} \mathrm{C}$ (an example heating profile for these MWH reactions can be seen in ESI Figure S3). This resulted in significantly higher conversions being delivered by both of these reactions (i.e. 72 and $81 \%$ respectively).

Again both $\mathrm{CH}$ and MWH heating results were very similar in both yield and product polymer molecular characteristics. Thus the only scenario that produced a differentiation between $\mathrm{CH}$ and $\mathrm{MWH}$ was at the high initiator 
loadings, which supports the idea that the greater initiator loading promotes the rapid increase in reaction temperature and rate via the selective heating of the radicals generated. In practice, the radical concentration has reached a sufficient level such that the energy input into the medium by the microwaves via the radicals is now contributing strongly to the heating processes within the system. Thus, this study has shown that microwaves are capable of delivering the sought after reaction time reductions provided the level of radicals present is sufficient to surpass the tipping point such that radical centred heating becomes a dominant mode of heat transfer in the system.

Consequently, this suggests that in any chemistry based system, if the species that is being selectively heated is present in too small a quantity, the predominant heat transfer method will remain as convection/conduction. Thus in such cases, there is no significant differentiated heating effect observed when MWH is compared to $\mathrm{CH}$ and so the outcomes of the reactions would be predicted to be similar. This critical concentration will be dependent on both the dielectric loss of the individual species and those of the other reaction components. Furthermore, as the dielectric loss has been shown to be nonlinear with temperature, then this concentration will also be dependent upon the reaction conditions utilised and will be dependent upon the power density achieved in the heated phase and the heat transfer coefficients of the system. Therefore, establishing an understanding of this connection is the subject of on-going study by the authors.

The initial temperature overshoot has also been used by the authors to accelerate the generation of catalyst via an 'in-situ' method. ${ }^{20}$ In these systems the same concentration effect has been observed. However, in this case it has 
been shown that the catalyst is selectively heating. However, in the case of 'insitu' CCTP it has proved to be difficult to accurately determine the dielectric properties of the organometallic species due to their low solubility. Additionally, the catalyst concentration is at the ppm level and from the conclusion drawn above this may be too small to have reached the threshold value which will deliver differentiated effects from the application of microwave heating. Especially when in the presence of another material which is significantly more susceptible to selective heating, in this case the radicals. ${ }^{16}$ Consequently, a series of experiments were conducted in which only $30 \mathrm{ppm}$ of $\mathrm{CoBr}_{2}$ utilising both $\mathrm{CH}$ and $\mathrm{MWH}$ both with and without pre-stirring. Furthermore, the AIBN concentration had been dropped back below the concentration that was noted to deliver the onset of volumetric heating (i.e. 0.5 wt $\%)$. Hence any differences should be linked to the efficiency of the catalyst formation process. The results from these experiments are shown in Table 6 .

Table 6. CCTP of MMA in the bulk using $30 \mathrm{ppm}$ of $\mathrm{CoBr}_{2}$ and dpg 'in-situ' catalyst and $0.5 \mathrm{wt} \%$ AIBN at $80^{\circ} \mathrm{C}$ for 2 hours

\begin{tabular}{cccccc}
\hline Entry & $\begin{array}{c}\text { Pre- } \\
\text { stirring }\end{array}$ & $\begin{array}{c}\text { Heating } \\
\text { Method }\end{array}$ & $\begin{array}{c}\text { Conv } \\
(\boldsymbol{\%})^{\mathbf{a}}\end{array}$ & PDI & $\begin{array}{c}\text { Mn } \\
\left(\mathbf{g ~ m o l}^{-\mathbf{1}}\right)^{\mathbf{b}}\end{array}$ \\
\hline $\mathbf{1}$ & Yes & CH & 88 & 2.78 & 2,800 \\
$\mathbf{2}$ & Yes & MWH & 89 & 2.36 & 2,500 \\
$\mathbf{3}$ & No & CH & 87 & 2.71 & 5,700 \\
$\mathbf{4}$ & No & MWH & 86 & 2.49 & 2,400 \\
a ${ }^{\text {determined by }}{ }^{1}$ H NMR. ${ }^{b}$ determined by GPC. & &
\end{tabular}

As expected, all reactions using $30 \mathrm{ppm}$ of the 'in-situ' catalyst exhibited a similar exotherm (see ESI Figure S3) which was attributed to a combination of the reduced retardation because of the drop in the CCTP agent concentration and the increased viscosity of the reaction mixture resulting from the higher 
molecular weight polymers being produced. It was observed that with this catalyst loading and allowing the typical standard exotherms to take place, produced high conversions (i.e. in all cases above 85\%) suggesting that both $\mathrm{CH}$ and $\mathrm{MWH}$ experiments should again produce similar polymer products. However, in both the experiments with and without pre-stirring, the MWH experiments were found to produce polymers with lower molecular weight and PDIs when compared with the $\mathrm{CH}$ equivalents (comparison of Table 6, Entries 1 with 2 and 3 with 4 ). The trend observed on the application of stirring at this concentration in the $\mathrm{CH}$ experiments is reversed. In this case the pre-stirring produced a significantly reduced molecular weight. This has been attributed to the increased dilution which has lead to a much slower formation of the CCTP catalyst attributed to diffusion issues, which are overcome by the introduction of stirring. Therefore the active catalyst cannot form quickly enough to effectively control the reaction, hence in the non-stirred $\mathrm{CH}$ experiment the polymer has a Mn of approximately double that of the stirred (Table 6, Entries 3 and 1 respectively). However, in the case of the microwave experiments there is no difference in the product of the experiment which are or are not prestirred, which indicated that there are no difference in the diffusion issues in these cases. Hence, it was concluded that the difference in the MWH reaction was related to the direct heating of specific molecular species within the mixture, which if present above a specific critical concentration, would result in differentiated level of temperature increases and reaction rate than $\mathrm{CH}$ systems. In this case, selective targeting of energy into the organometallic species, aids in the formation of the catalyst, allowing it to form rapidly even at this low concentration and can, therefore, control the reaction more effectively. 
This conclusion is supported by the fact that both stirred and non-stirred reaction exhibit smaller PDI's that the $\mathrm{CH}$ heating. These PDI's are higher than 2 because of the viscosity of the reaction medium at the high conversions achieved, which again suggests that the microwave reactions are under better control that the $\mathrm{CH}$ equivalents. This is because the $\mathrm{MWH}$ are more successfully overcoming the diffusion in the high viscosity, high conversion reaction mixtures and because the catalyst is present in the expected quantities having been efficiently formed as a result of selective heating of the precursors.

\section{Conclusion}

This study clearly demonstrated that efficient CCTP control has been achieved using CCTP agents derived from $\mathrm{CoCl}_{2}, \mathrm{CoBr}_{2}$ and $\mathrm{CoI}_{2}$ precursors, provided a single stage, 'in-situ' catalyst preparation method was adopted. Consequently, these developments fulfil several of the Principles of Green Chemistry (PGC). Removing the need to pre-synthesise catalyst reduces catalyst degradation and prevents additional waste (PGC 1), improves the atom efficiency of both catalyst synthesis and polymerisation (PGC 7) and removes the need for organic solvents (PGC 8). Empirically, the order of catalyst activity was shown to be $\mathrm{Br}>\mathrm{Cl}>\mathrm{I}$ with MMA, which is a result of the combined influence of complex stability, solubility and ease of synthesis. However, the use of a less sterically crowded equatorial ligand was shown to deliver a reduction in efficiency increase at high catalyst concentrations. Hence, the optimum catalyst system within this series for the CCTP of MMA was concluded to be the 'in-situ' preparation/use of dibromo $\left(\operatorname{dpgH}_{2}\right)_{2}$ cobalt. 
Additionally, routes to achieve reductions in the 'in-situ' reaction cycle times have been successfully defined. At high catalyst concentration $(\sim 600 \mathrm{ppm})$ removing pre-stirring reduced processing time by 1 hour, whilst producing a better quality polymer product. Pre-stirring was concluded to introduce greater levels of catalyst deactivation prior to polymerisation. However, at lower concentrations (approximately $30 \mathrm{ppm}$ ) and in $\mathrm{CH}$ systems pre-stirring was required to overcome the additional dilution of the precursors. Subsequently, the application of microwave heating to such reaction systems, which contained a specific initiator concentration range $(<1 \mathrm{wt} \%)$, resulted in a process which exhibited faster system heat up times, did not require prestirring and resulted in a high yield $(>80 \%)$ of the target polymer. This was attributed to selective microwave heating overcoming diffusion barriers at low catalyst pre-cursor concentrations. Hence, optimisation of the cycle time and the consequent reduced agitation/heating duty for the polymerisation, when combined with the removal of the entire catalyst production process was shown to deliver a significant increase in CCTP energy efficiency (PGC 9). Consequently, the ability to more improve production strategies which utilise "in-situ" catalyst manufacture represents a real process benefit which may underpin the adoption of microwave heating strategies at the commercial scale.

Finally, it has been concluded that, for any particular reaction system, there is a critical concentration of a selective heating species which is required if MWH is to produce differentiated effects to $\mathrm{CH}$ processes. This will be dependent on combination of factors including dielectric properties, relative concentrations and reaction conditions applied.

\section{References}


1. P. Haque, I. A. Barker, A. Parsons, K. J. Thurecht, I. Ahmed, G. S. Walker, C. D. Rudd and D. J. Irvine, Journal of Polymer Science Part aPolymer Chemistry, 2010, 48, 3082-3094.

2. J. Charles E. Carraher, Editon edn., 2000.

3. G. D. Airey, J. Wilmot, J. R. A. Grenfell, D. J. Irvine, I. A. Barker and J. El Harfi, European Polymer Journal, 2011, 47, 1300-1314.

4. J. Li, J. El Harfi, S. M. Howdle, K. Carmichael and D. J. Irvine, Polymer Chemistry, 2012, 3, 1495-1501.

5. K. J. Abbey, D. L. Trumbo, G. M. Carlson, M. J. Masola and R. A. Zander, Journal of Polymer Science Part a-Polymer Chemistry, 1993, 31, 3417-3424.

6. P. Cacioli, D. G. Hawthorne, R. L. Laslett, E. Rizzardo and D. H. Solomon, Journal of Macromolecular Science-Chemistry, 1986, A23, 839-852.

7. J. F. Morizur, D. J. Irvine, J. J. Rawlins and L. J. Mathias, Macromolecules, 2007, 40, 8938-8946.

8. D. M. Haddleton, C. Topping, D. Kukulj and D. Irvine, Polymer, 1998, 39, 3119-3128.

9. A. A. Gridnev and S. D. Ittel, Chemical Reviews, 2001, 101, 3611-3659.

10. J. P. A. Heuts and N. M. B. Smeets, Polymer Chemistry, 2011, 2, 24072423.

11. W. X. Wang, P. A. Stenson, A. Marin-Becerra, J. McMaster, M. Schroder, D. J. Irvine, D. Freeman and S. M. Howdle, Macromolecules, 2004, 37, 6667-6669.

12. Schrauze.Gn and Windgass.Rj, Journal of the American Chemical Society, 1966, 88, 3738-\&.

13. A. A. Gridnev, Polymer Journal, 1992, 24, 613-623.

14. K. G. Suddaby, D. M. Haddleton, J. J. Hastings, S. N. Richards and J. P. Odonnell, Macromolecules, 1996, 29, 8083-8091.

15. K. Adlington, A. Green, W. X. Wang, S. M. Howdle and D. J. Irvine, Dalton Transactions, 2013, 42, 127-136.

16. K. Adlington, G. J. Jones, J. El Harfi, G. Dimitrakis, A. Smith, S. W. Kingman, J. P. Robinson and D. J. Irvine, Macromolecules, 2013, 46, 3922-3930.

17. N. Dubois, D. Glynn, T. McInally, B. Rhodes, S. Woodward, D. J. Irvine and C. Dodds, Tetrahedron, 2013, 69, 9890-9897.

18. C. Guerrero-Sanchez, M. Lobert, R. Hoogenboom and U. S. Schubert, Macromolecular Rapid Communications, 2007, 28, 456-464.

19. C. Guerrero-Sanchez, R. Hoogenboom and U. S. Schubert, Chemical Communications, 2006, 3797-3799.

20. N. T. Nguyen, E. Greenhalgh, M. J. Kamaruddin, J. El harfi, K. Carmichael, G. Dimitrakis, S. W. Kingman, J. P. Robinson and D. J. Irvine, Tetrahedron, 2014, 70, 996-1003

21. S. L. Dong, R. Padmakumar, R. Banerjee and T. G. Spiro, Inorganica Chimica Acta, 1998, 270, 392-398.

22. K. P. Jensen, S. P. A. Sauer, T. Liljefors and P. O. Norrby, Organometallics, 2001, 20, 550-556.

23. K. G. Suddaby, D. R. Maloney and D. M. Haddleton, Macromolecules, 1997, 30, 702-713.

24. P. A. Clay and R. G. Gilbert, Macromolecules, 1995, 28, 552-569. 
Page $\mathbf{2 8}$ of $\mathbf{2 8}$ 